\title{
Aplicación de un modelo de Historia Clínica Perinatal
}

\author{
EN EL HOSPITAL UNIVERSITARIO DEL VALLE
}

\author{
Dr. Edgar Cobo
}

Ha llegado la hora de ayudar

a rehacer la vida sobre muertos,

a sacar pan de los que están dormidos

y corazón de los que están despiertos

Carlos Castro Saavedra

"Entrad en la esperanza. . ."

La historia de la Historia Clínica Perinatal (HCP) se inicia en nuestro país en 1979, cuando en reunión con un grupo de entusiastas gineco-obstetras de Medellín, decidimos impulsar el diseño de HCP propuesto por el Centro Latinoamericano de Perinatología y Desarrollo Humano (CLAP), ubicado en Montevideo (1). No veíamos otra salida al deseo frustrado durante tantos años, de conocer científicamente la realidad de la salud materno-infantil en los Hospitales Universitarios del país. Este modelo había sido incorporado por algunos Departamentos de Obstetricia y Ginecología en América Latina, habiendo llegado solo uno a la etapa de diagnóstico descriptivo de la situación perinatal, con sistematización de la información (2). Se inició entonces la implantación de la HCP con

Profesor Titular. Departamento de Obstetricia y Ginecología. Universidad del Valle, Cali. Colombia.

Proyecto financiado con una donación de la Fundación para la Educación Superior (FES). la asesoría del CLAP, dando como resultado avances notables en la exploración de la situación perinatal en Medellín $(3,4)$ y en Cali.

En 1982, la Federación Colombiana de Sociedades de Obstetricia y Ginecología y la sección Materno-Infantil del Ministerio de Salud suscribieron un contrato a través del cual esta última, compromete la asesoría de la Federación para la reforma de la HCP y el diseño de estrategias para su implantación, enmarcandola dentro del objetivo de una historia clínica única del Ministerio de Salud, para todo el país. La comisión nombrada para este propósito, y de la cual nosotros no hacemos parte, ha realizado ya la reforma de la HCP e inicia el proceso de estrategias de implantación y de sistematización de la información obtenida.

El autor no ha considerado como material de publicación científica este tipo de informes, los cuales ha distribuido como documentos de circulación intrainstitucional dentro del Hospital y el Sistema Seccional de Salud. Sin embargo, el alto número de consultas del profesorado de Obstetricia y Ginecología, de Pediatría y de personal de la Seccional de Salud del Valle, a este documento, así como la frecuente referencia al mismo en reuniones locales y nacionales $y$ 
además, una cierta presión intrahospitalaria sobre la conveniencia de una divulgación más amplia de estos datos, han llevado este informe hacia el inesperado destino de la publicación formal.

El informe corresponde al período comprendido entre el 10 . de marzo de 1982 y el 28 de febrero de 1983 , durante el cual se realizó la aplicación formal de la HCP, que venía probándose desde julio de 1981. Contiene datos alarmantes sobre la realidad de la atención maternoinfantil, administrada a una población extremadamente patológica y en consecuencia, merecedora de un alto nivel profesional y técnico dentro de la institución.

Como resultado de este estudio hemos avanzado en el diseño de una serie de normas que buscan mejorar la atención de la patología observada y planteamos de nuevo una modificación estructural de la actividad de supervisión del personal docente sobre el manejo de tan abundante patología, basándonos en la dificultad (casi la imposibilidad) de aumentar significativamente los recursos humanos existentes. No sabemos aún, si con estas reformas podamos quebrar las altas tasas de morbilidad y mortalidad que se describen adelante. Pero sabemos que la actividad que no deberá interrumpirse nunca, es la del perfeccionamiento del método de registro $y$ la sistematización del análisis de los datos.

No tengo dudas al afirmar que dentro de las condiciones actuales no es posible atender adecuadamente a la población caracterizada en este estudio. Estamos haciendo lo posible, pero la realidad nos va empujando hacia lo imposible. Ese es el desafío.

\section{PACIENTES Y METODOS}

En el período estudiado se atendieron 8.181 pacientes hospitalizadas, cuyas historias clínicas perinatales (HCP) son el material de este informe. La HCP fue elaborada por estudiantes de pregrado, quienes recibieron información teórica $y$ práctica sobre la metodología antes de iniciar su trabajo. Antes del alta de las pacientes se llenó el resumen estadístico perinatal (REP) para cada paciente, durante los días hábiles. No fue posible hacer lo mismo durante los fines de semana y los días feriados, en los cuales se hizo el REP después del alta de las pacientes. El REP consta de 80 variables precodificadas. Tanto el volcado, o reducción de los datos de la HCP al REP, como el contaje manual de todos los datos, fue realizado por 3 auxiliares de registros médicos, cada mes. La reducción manual de los datos a cuadros y figuras, la supervisión periódica del proceso, la síntesis anual, el cálculo y ordenamiento de las frecuencias relativas y el análisis que se presenta, fueron realizados por el autor de este informe.

\section{RESULTADOS}

En un estudio en el cual tenemos como meta la obtención de datos "du-

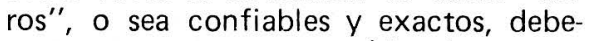
mos comenzar por una crítica de nuestro trabajo. En los datos hay subregistro y alguna proporción de inconsistencias numéricas, lo cual plantea la necesidad de contratar personal diferente, más capacitado $y$ en número mayor al actual, para el trabajo futuro. Si se tiene en cuenta, sin embargo, que el proceso total significa la captación y el registro manual de varios millones de datos, podría aceptarse que este primer año deja datos bastante aproximados, ya que la pérdida de información está alrededor del $4 \%$, fluctuando entre 0 y $16.1 \%$, según el parámetro estudiado. (Cuadro No. 1).

Los datos actuales constituyen una línea de base y pertenecen a una fase descriptiva básica de los eventos ocurridos en nuestro Servicio. En los próximos años tendrá que venir un análisis completo de las múltiples variables correlacio- 
nadas entre sí, realizado con un programa de computación diseñado específicamente para este trabajo.

\section{Cuadro No. 1}

PORCENTAJE DE FALLAS

EN LA CONFECCION DE LA HCP

Y EN EL VOLCADO Y CONTAJE DE LOS DATOS (REP)

Estado Civil

Edad Materna

Instrucción de la Embarazada

Ocupación de la Embarazada

Ocupación del cónyuge

Vivienda del Cónyuge

Gestaciones Previas

Hábito de Fumar

Intervalo Intergenésico

Peso anterior al Embarazo

Talla Materna

Edad Gestacional a la $1^{\text {a }}$ Consulta

Númiero de Controles Prenatales

Presentación

$N^{\circ}$ de Fetos

Patología del Enbarazo

Edad Gestacional al Parto

Iniciación del Parto

Anestesia

Terminación del Parto

Sexo del Recién Nacido

Tiempo RM-Parto

Días de Hospitalización

Indice de Apgar $1 \mathrm{~min}$.

Indice de Apgar 5 min.

Personal que atendió al R.Nacido

Peso del R.N.

Patología Neonatal

Puerperio

\begin{tabular}{|c|c|}
\hline $\mathrm{HCP}$ & REP \\
\hline 16.1 & 0 \\
\hline 0.1 & 0.4 \\
\hline 1.7 & 0.6 \\
\hline 1.4 & 0 \\
\hline 13.1 & - \\
\hline 3.5 & 0 \\
\hline 2.0 & 16.3 \\
\hline 1.1 & - \\
\hline 4.2 & 0 \\
\hline 3.1 & 0.2 \\
\hline - & - \\
\hline - & - \\
\hline 8.8 & 0 \\
\hline 9.0 & 0 \\
\hline 0.5 & 4.6 \\
\hline 0.5 & 4.6 \\
\hline 0 & 0 \\
\hline 0.7 & 7.1 \\
\hline 2.3 & 0.3 \\
\hline 0.1 & 1.0 \\
\hline 0 & 9.1 \\
\hline 4.3 & 3.1 \\
\hline 14.5 & 3.5 \\
\hline- & - \\
\hline 1.3 & 9.3 \\
\hline 0.9 & 10.0 \\
\hline 1.2 & 9.5 \\
\hline 1.1 & 10.0 \\
\hline- & 9.2 \\
\hline 0 & 4.2 \\
\hline
\end{tabular}

Instrucción del Cónyuge

\section{Caracterización Socio-Económica de la población atendida}

El análisis de los cuadros 2 a 8 muestra una población con factores de riesgo propios del nivel económico al que pertenece. Sin entrar en un detalle pormenorizado que se deja intencionalmente a cargo de los lectores del informe, pueden citarse como ejemplos que sólo 1 de cada 5 mujeres atendidas tienen una relación legal con su cónyuge; que la
Cuadro No. 2

ESTADO CIVIL

\begin{tabular}{|l|r|c|}
\multicolumn{1}{c}{} & $n$ & $\%$ \\
\hline Unión Libre & 3.816 & 46.6 \\
\hline Casadas & 1.750 & 21.4 \\
\hline Solteras & 1.254 & 15.3 \\
\hline Separadas & 32 & 0.4 \\
\hline Viudas & 11 & 0.14 \\
\hline Divorciadas & 3 & 0.04 \\
\hline No Info & 1.315 & 16.1 \\
\hline
\end{tabular}

In

8.181

Cuadro No. 3

EDAD MATERNA

$\%$

\begin{tabular}{|l|r|r|}
\hline$<15$ & 54 & 0.7 \\
\hline $15-19$ & 1.945 & 23.9 \\
\hline $20-24$ & 2.709 & 33.2 \\
\hline $25-29$ & 1.619 & 19.9 \\
\hline $30-34$ & 991 & 12.2 \\
\hline $35-39$ & 607 & 7.4 \\
\hline $40-44$ & 191 & 2.3 \\
\hline$>45$ & 22 & 0.3 \\
\hline No Info & 11 & 0.1 \\
\hline
\end{tabular}

$\sum n$

8.149

Cuadro No. 4

INSTRUCCION DE LA EMBARAZADA

\begin{tabular}{|l|r|r|}
\hline \multicolumn{2}{c}{$n$} & n \\
\hline Analfabeta & 423 & 5.2 \\
\hline Primaria Incompleta & 2.784 & 34.3 \\
\hline Primarid Completa & 1.799 & 22.1 \\
\hline Secundaria Incompleta & 2.637 & 32.4 \\
\hline Secundaria Conpleta & 263 & 3.2 \\
\hline Universitaria Incompleta & 78 & 0.9 \\
\hline Universitaria Completa & 12 & 0.1 \\
\hline No Info & 129 & 1.7 \\
\hline
\end{tabular}

$\Sigma n$

8.125 
cuarta parte es menor de 20 años; que la casi totalidad $(94 \%)$ no ha completado el segundo ciclo de educación básica; que cerca del $90 \%$ no tiene trabajo remunerado y que la mitad de esa población debe compartir su habitación con dos o más personas. Se destaca además, que entre los cónyuges declarados, si bien se observa una muy ligera ventaja en la educación (75\% sin completar el segundo ciclo), hay un 5 \% de desocupados y un $30 \%$ sin ocupación estable.

Cuadro No. 5

INSTRUCCION DEL CONYUGE

\begin{tabular}{|l|r|r|}
\multicolumn{2}{c}{} & \multicolumn{1}{c}{$n$} \\
\hline Analfabqta & 202 & 2.9 \\
\hline Primaria Incompleta & 1.496 & 21.7 \\
\hline Primaria Completa & 1.347 & 19.5 \\
\hline Secundaria Incompleta & 2.153 & 31.3 \\
\hline Secundaria Completa & 552 & 8.0 \\
\hline Universitaria Incompleta & 201 & 2.9 \\
\hline Universitaria Completa & 36 & 0.5 \\
\hline No Info & .904 & 13.1 \\
\hline
\end{tabular}

Cuadro No. 6

OCUPACION DE LA EMBARAZADA

NO REMUNERADA

\begin{tabular}{|l|r|r|}
\hline Hogar, Sin ayuda & 5.961 & 72.9 \\
\hline Hogar, Con ayuda & 1.707 & 20.9 \\
\hline No trabaja & 396 & 4.8 \\
\hline No Info & 115 & 1.4 \\
\hline
\end{tabular}

REMUNERADA

\begin{tabular}{|}
\hline$<4$ horas, con predominio de actividad fisica & 232 & 2.9 \\
\hline$>4$ horas, con predominio de actividad fisica & 657 & 8.1 \\
\hline$<4$ horas, con predominio de actividad psiquica & 6 & 0.1 \\
\hline$>4$ horas, con predominio de actividad psiquica & 22 & 0.3 \\
\hline Sin Trabajo Remunerado & 7.065 & 87.2 \\
\hline No Info & 115 & 1.4 \\
\hline
\end{tabular}

$\Sigma n=8.097$
Cuadro No. 7

OCUPACION DEL CONYUGE

\begin{tabular}{|l|r|r|}
\hline Sin Ocupación & 340 & 4.9 \\
\hline Obrero No Estable & 2.028 & 29.5 \\
\hline Obrero Estable & 2.208 & 32.1 \\
\hline Einpleado o Comerciante & 1.713 & 24.9 \\
\hline Protesional o Técnico & 351 & 5.1 \\
\hline No Info & 239 & 3.5 \\
\hline
\end{tabular}

In

6.879

Cuadro No. 8

VIVIENDA

No. DE PERSONAS POR HABITACION

n

\begin{tabular}{|l|r|r|}
\hline 1 & 733 & 9.1 \\
\hline 2 & 3.477 & 43.2 \\
\hline 3 & 1.940 & 24.1 \\
\hline 4 & 843 & 10.5 \\
\hline$>5$ & 897 & 11.1 \\
\hline No Info & & 2.0 \\
\hline
\end{tabular}

$\sum n$

8.050

\section{Gestaciones previas}

La mayoría de la población atendida está compuesta por primigrávidas (38.5\%). $\mathrm{Si}$ a ella se suman las pacientes con uno o dos embarazos previos se observa que el $73 \%$ de la población atendida tiene familias pequeñas.

\section{Hábito de fumar}

El $83 \%$ de las pacientes no fuma, lo cual constituye un hábito sanitario importante en términos de bajo peso al nacer. No sería necio estudiar si esta cualidad de nuestras pacientes tiene fundamentos sanitarios, lo cual parece im- 
probable dado su nivel educacional, o si sus fundamentos son económicos, 10 cual parece casi evidente.

\section{Cuadro No. 9}

GESTACIONES PREVIAS

\begin{tabular}{|c|c|c|}
\hline & $n$ & $\%$ \\
\hline 0 & 3.150 & 38.5 \\
\hline 1 & 1.674 & 20.5 \\
\hline 2 & 1.141 & 13.9 \\
\hline 3 & 742 & 9.1 \\
\hline 4 & 458 & 5.6 \\
\hline$>5$ & 922 & 11.3 \\
\hline No Info & 92 & 1.1 \\
\hline
\end{tabular}

Cuadro No. 10

HABITO DE FUMAR

n $\%$

\begin{tabular}{|l|r|r|}
\hline No & 6.743 & 82.4 \\
\hline $1-5 /$ dia & 872 & 10.6 \\
\hline $6-10$ & 150 & 1.8 \\
\hline $11-15$ & 19 & 0.2 \\
\hline $16-20$ & 58 & 0.7 \\
\hline$>21$ & 7 & 0.1 \\
\hline No Info & 332 & 4.2 \\
\hline
\end{tabular}

$\sum n$

8.181

\section{Intervalo intergenesico}

$\mathrm{Si}$ se excluye las primigrávidas, se observa que la mitad de las pacientes tienen intervalos intergenésicos iguales o mayores a 2 años. En contraste, una proporciói relativamente alta (15\%) inicia un nuevo embarazo en los primeros 5 meses después del parto anterior.
Cuadro No. 11

INTERVALO INTERGENESICO

Meses
\begin{tabular}{|l|c|}
\hline$x$ Primi & $3.145(38.5 \%)$ \\
\hline $0-5$ & 711 \\
\hline $6-11$ & 563 \\
\hline $12-17$ & 902 \\
\hline $18-23$ & 162 \\
\hline ¿4 & 2.426 \\
\hline NO INF & $252(3.1 \%)$ \\
\hline En & 8.161 \\
\hline
\end{tabular}

con intervalo $(n=4.764)$

\begin{tabular}{|c|}
\hline$\%$ \\
\hline 14.9 \\
\hline 11.8 \\
\hline 18.9 \\
\hline 3.4 \\
\hline 51.0 \\
\hline
\end{tabular}

\section{Peso anterior al embarazo actual}

Este es un dato de díficil obtención según se ha observado en este estudio $y$ estudios similares realizados en fmérica Latina. Al parecer el $30 \%$ de las madres no conoce su peso anterior al embarazo. De todas maneras, vale la pena destacar que el $21.5 \%$ de las madres presentó pesos iguales o menores a $50 \mathrm{~kg}$, lo cual constituye factor de riesgo para parto prematuro y bajo peso para la edad gestacional (Desnutrición Intrauterina).

\section{Cuadro No. 12}

PESO ANTERIOR

\begin{tabular}{|c|c|c|} 
Kg. & n & \% \\
\hline $34-40$ & 143 & 1.9 \\
\hline $41-45$ & 487 & 6.4 \\
\hline $46-50$ & 1.008 & 13.2 \\
\hline $51-55$ & 1.010 & 13.2 \\
\hline $56-60$ & 1.386 & 18.1 \\
\hline $61-65$ & 608 & 7.9 \\
\hline $66-70$ & 335 & 4.4 \\
\hline $71-75$ & 213 & 2.7 \\
\hline $76-80$ & 122 & 1.6 \\
\hline $81-85$ & 31 & 0.4 \\
\hline $86-90$ & 18 & 0.3 \\
\hline $91-95$ & 13 & 0.1 \\
\hline $96-100$ & 12 & 0.7 \\
\hline NO INF0 & 2.281 & $29.7^{\star}$ \\
\hline En & 7.667 & \\
\hline
\end{tabular}




\section{Talla materna}

El $18 \%$ presenta tallas iguales o menores a $1.49 \mathrm{mt}$. lo cual aumenta la probabilidad de desproporción fetopélvica.

\section{Cuadro No. 13}

TALLA MATERNA

\begin{tabular}{|c|c|c|}
\multicolumn{1}{c}{$\mathrm{mt}$} & $\mathrm{n}$ & \% \\
\hline $1.30-1.34$ & 13 & 0.2 \\
\hline $1.35-1.39$ & 60 & 0.7 \\
\hline $1.40-1.44$ & 371 & 4.5 \\
\hline $1.75-1.49$ & 1.049 & 12.8 \\
\hline $1.50-1.54$ & 1.970 & 24.1 \\
\hline $1.55-1.59$ & 1.986 & 24.3 \\
\hline $1.60-1.64$ & 1.265 & 15.5 \\
\hline $1.65-1.69$ & 420 & 5.1 \\
\hline $1.70-1.74$ & 87 & 1.1 \\
\hline $1.75-1.79$ & 34 & 0.4 \\
\hline 1.80 & 1 & - \\
\hline NO INFO & 925 & 11.3 \\
\hline
\end{tabular}

$\Sigma \mathrm{n}$

8.181

\section{Edad gestacional a la primera consulta prenatal}

Se destaca que 1 de cada 4 pacientes no tiene control prenata! ni en nuestra Institución, ni fuera de ella, lo cual constituye una cifra extremadamente alta. La mayoría de las pacientes que sí lo tuvieron (60\%) consultaron precozmente, o sea en los dos primeros trimestres del embarazo. La mayoría tuvo entre 4 y 6 consultas de control. (Cuadro No. 15).

\section{Presentación y número de fetos}

Estos datos corresponden con lo observado generalmente en los servicios de obstetricia y de ellos nos interesa especialmente la proporción de presentaciones podálicas $(9 \%$ y de embarazos múltiples (2\%), ya que entre ellos podría existir patología evitable mediante la implantación de normas institucionales de atención. (Véanse cuadros 16 y 17).

Cuadro No. 14

EDAD GESTACIONAL A LA PRIMERA CONSULTA PRENATAL

\begin{tabular}{|c|c|c|}
\hline meses & $n$ & \% \\
\hline$N I N G \cup N A$ & 1.973 & 24.1 \\
\hline 1 & 718 & 8.8 \\
\hline 2 & 973 & 11.9 \\
\hline 3 & 1,092 & 13.4 \\
\hline 4 & 979 & 12.0 \\
\hline 5 & 621 & 7.6 \\
\hline 6 & 509 & 6.3 \\
\hline 7 & 356 & 4.3 \\
\hline 8 & 217 & 2.6 \\
\hline 9 & 18 & 0.2 \\
\hline NO INFO & 720 & 8.8 \\
\hline
\end{tabular}

Cuadro No. 15

NUMERO DE CONTROLES PRENATALES

\begin{tabular}{|c|c|c|}
\hline 0 & 1.973 & 24.1 \\
\hline 1 & 157 & 1.9 \\
\hline 2 & 454 & 5.6 \\
\hline 3 & 663 & 8.1 \\
\hline 4 & 950 & 11.6 \\
\hline 5 & 903 & 11.1 \\
\hline 6 & 975 & 11.9 \\
\hline 7 & 525 & 6.4 \\
\hline 8 & 420 & 5.1 \\
\hline 9 & 425 & 5.2 \\
\hline No Inf $\rho$ & 734 & 9.0 \\
\hline
\end{tabular}

$\sum n$

8.179

\section{Patología en embarazo}

En este parámetro no hubo pérdida significante de datos, ni en la elaboración 
de la historia, ni en el volcado de la misma al resumen estadístico perinatal, lo cual hace su estudio confiable.

\section{Cuadro No. 16}

PRESENTACION

\begin{tabular}{|l|r|r|}
\multicolumn{2}{c}{} & \multicolumn{1}{c}{$n$} \\
\hline Cefálica & 6.961 & 89.2 \\
\hline Podálica & 733 & 9.4 \\
\hline Transversa & 73 & 0.9 \\
\hline No Info & 40 & 0.5 \\
\hline
\end{tabular}

$\Sigma n$

7.807

Cuadro No. 17

NUMERO DE FETOS

\begin{tabular}{|c|r|c|}
\hline 1 & 7.598 & 97.3 \\
\hline 2 & 165 & 2.1 \\
\hline 3 & 1 & 0.01 \\
\hline No Info & 40 & 0.5 \\
\hline
\end{tabular}

$\sum n$

7.807

Se observa en primer lugar que la gran mayoría de los casos (79\%), presenta patología durante el embarazo. Esta cifra está aumentando ya que en un estudio interno realizado en 1978 era del $62 \%$ Posiblemente esta concentración de patología sea el resultado de una razonable utilización del sistema de referencia de pacientes desde la periferia. $\mathrm{Si}$ agregáramos en este estudio factores de riesgo, como: edad, paridad, educación, peso y talla, posiblemente la totalidad de la muestra podría ser clasificada como de al to riesgo obstétrico.

La distribución de la patología sigue las proporciones características de un país subdesarrollado (Infección, Hiper- tensión y Hemorragia), pero con algunas particularidades, que se destacan a continuación:

a) Una elevada e inexplicable proporción de rotura prematura de las membranas

Cuadro No. 18

PATOLOGIA DEL EMBARAZO

\begin{tabular}{|l|r|r|}
\hline Con Patología & 6.461 & 79.0 \\
\hline Sin Patología & 1.717 & 21.0 \\
\hline No Info & 3 & \\
\hline In & 8.181 \\
\end{tabular}

n $\hbar^{*}$

INFECCION
\begin{tabular}{|l|r|r|}
\hline Infección Urinaria & 893 & 10.9 \\
\hline Aborto Infectado & 412 & $\mathbf{5 . 0}$ \\
\hline Sepsis Puerperal & 252 & $\mathbf{3 . 1}$ \\
\hline Avinionitis & 126 & 1.5 \\
\hline TBC Pulmonar & 35 & 0.4 \\
\hline Paludismo & 3 & - \\
\hline Otras & 88 & 1.1 \\
\hline
\end{tabular}

2. ROTURA PREMATURA DE MEMBRANAS $\quad 1.774 \quad 21.7$

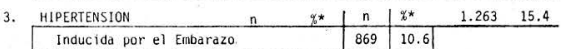

\begin{tabular}{|c|c|c|c|c|}
\hline \multicolumn{3}{|c|}{ Inducida por el Embarazo. } & 869 & 10.6 \\
\hline -Preclampsia Leve & 233 & 2.8 & \multirow[b]{4}{*}{394} & \multirow{5}{*}{4.8} \\
\hline - freclampsia Severa & 590 & 7.2 & & \\
\hline -Eclampsia & 46 & 0.6 & & \\
\hline \multicolumn{3}{|l|}{ Crónica } & & \\
\hline -Con I.P.E. Agregada & 50 & 0.6 & & \\
\hline
\end{tabular}

- sobre las 8.181 pacientes

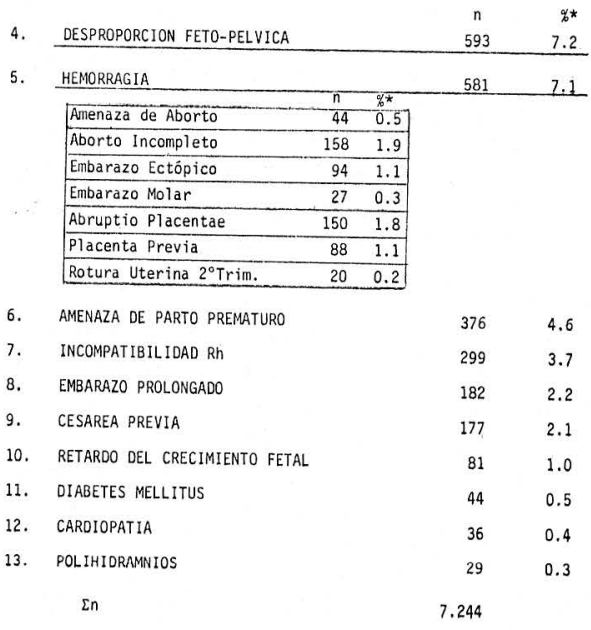

* sobre las 8.181 pacientes 
ovulares, aparece ocupando el segundo lugar $(21.7 \%$ ) entre la patología observada. Este dato merece un estudio más detallado que nos permita ubicar tanto la procedencia de estos casos como sus posibles causas, ya que podría existir un componente iatrogénico. La proporción de esta patología es menor del $10 \%$ en la mayoría de las maternidades consul. tadas.

b) La infección ocupa el primer lugar y entre ellas ocupan los primeros lugares: la infección urinaria (11\% del total de la patología) el aborto séptico (5\%) y la sepsis puerperal (3\%).

Han reaparecido casos de paludismo.

c) La hipertensión arterial, bien sea inducida por el embarazo (toxemia) o bien sea crónica, parece conservar proporciones similares a las cuantificadas ocasionalmente en los últimos 20 años.

d) Entre las hemorragias sigue ocupando un lugar destacado el aborto. Estos datos sólo se refieren a aquellos casos que requieren hospitalización, ya que la mayoría de los casos se maneja ambulatoriamente.

e) Existe una elevada proporción de desprendimiento prematuro de la placenta normalmente insertada. En el Hospital Pereira-Rossel de Montevideo y en la década del 50, aparecia un caso por año $y$ en esta serie observamos un caso cada $21 / 2$ días.

f) Se pone en evidencia una elevada pro. porción de roturas uterinas. Este es un indicador muy sensible de calidad de atención obstétrica y está prácticamente eliminado de los servicios modernos de obstetricia. Entre nosotros se registran aproximadamente dos roturas uterinas por mes. Se estudiará con mayor detalle para deslindar aquellos casos que ocurrieron fuera de la Institución, ya que es un dato fundamental de auditoría médica.
Gráfico No. 1

PATOLOGIA MATERNA

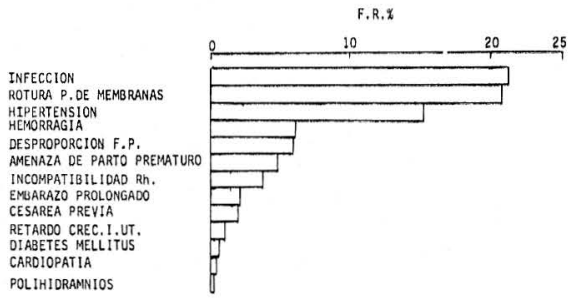

Cuadro No. 19

EDAD GESTACIONAL AL PARTO O ABORTO

Semanas

\begin{tabular}{|r|r|r|}
\hline$<26$ & 126 & 1.7 \\
\hline 26 & 20 & 0.2 \\
\hline 27 & 19 & 0.2 \\
\hline 28 & 44 & 0.6 \\
\hline 29 & 9 & 0.1 \\
\hline 30 & 74 & 0.9 \\
\hline 31 & 34 & 0.5 \\
\hline 32 & 105 & 1.4 \\
\hline 33 & 49 & 0.6 \\
\hline 34 & 128 & 1.7 \\
\hline 35 & 139 & 1.8 \\
\hline 36 & 342 & 4.5 \\
\hline 37 & 351 & 4.6 \\
\hline 38 & 783 & 10.3 \\
\hline 39 & 857 & 11.4 \\
\hline 40 & 3.159 & 41.6 \\
\hline 41 & 526 & 6.9 \\
\hline 42 & 480 & 6.3 \\
\hline 43 & 186 & 2.5 \\
\hline 44 & 111 & 1.5 \\
\hline No & 57 & 0.7 \\
\hline
\end{tabular}

$\sum n$

7.599 
Gráfico No. 2

EDAD GESTACIONAL EN EL MOMENTO DEL PARTO

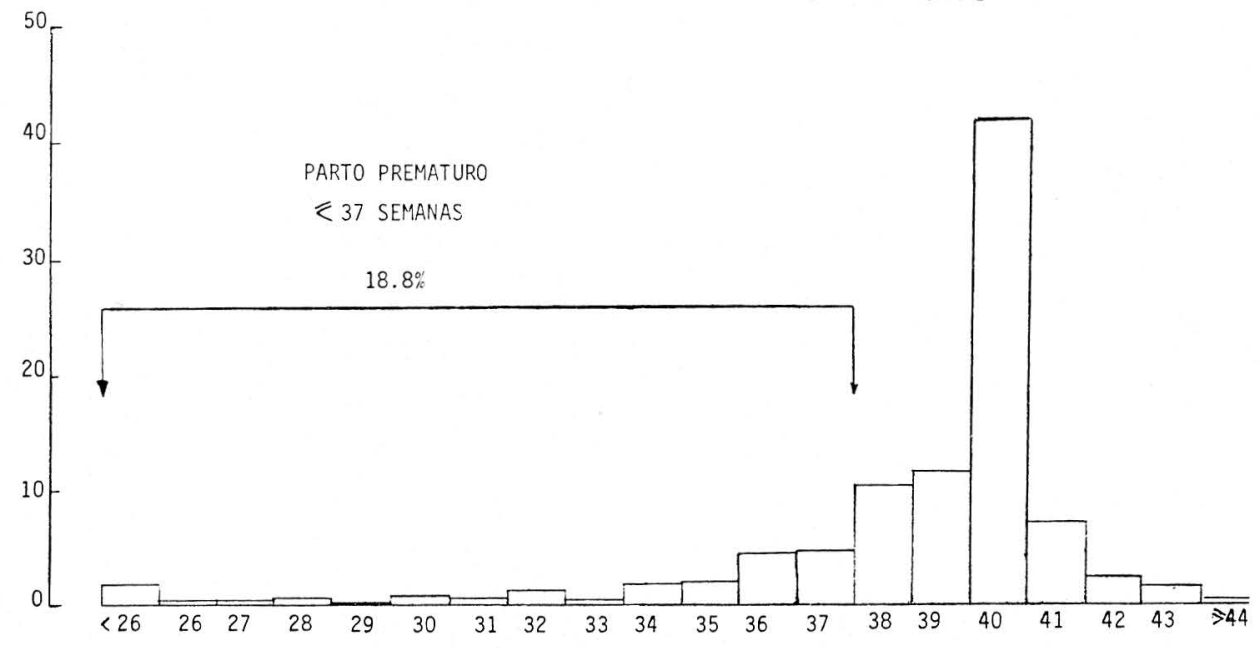

Edad gestacional en el momento del parto

Como hecho importante debe destacarse que el $19 \%$ de los nacimientos ocurre prematuramente, o sea, antes de la semana 38.

No conocemos la proporción exacta de casos en los cuales la prevención del parto prematuro sea posible dentro de la Institución, ya que solo el $4.6 \%$ de los casos atendidos (Cuadro No. 18) tuvieron el diagnóstico de amenaza de parto prematuro. Sin embargo, tenemos la impresión de que la mayoría de estos casos puede ser prevenida mediante la supervisión estricta de las normas del programa hospitalario de prevención de parto prematuro.

Una proporción de partos prematuros tiene indicación médica de interrupción del embarazo antes del término (toxemia, hemorragia, infección, etc.). Como muchos otros de los parámetros en estudio, éste exige diferentes cruces para establecer cuales son los casos evitables aplicando el programa de prevención mencionado.

Alrededor del $2 \%$ de los casos muestra diagnósticos de embarazos prolongados de los cuales, solo la decima parte corresponde a fetos postmaduros.

Cuadro No. 20

FORMA DE INICIACION DEL PARTO

\begin{tabular}{|l|r|r|}
\hline Espontáneco & 7.173 & 88.0 \\
\hline Inducido & 660 & 8.0 \\
\hline Estimulado & 137 & 1.7 \\
\hline No INFO & 186 & 2.3 \\
\hline in & 8.156 & \\
\hline
\end{tabular}

\section{Forma de iniciación del parto}

Aproximadamente el $90 \%$ de los partos se inició espontáneamente y el $10 \%$ fue inducido o estimulado. Estas cifras muestran una cierta tendencia interven- 
cionista en nuestro servicio si se los compara con los datos informados en otras maternidades de América Latina $y$ de nuestro país.

\section{Cuadro No. 21}

ANESTESIA PARA EL PARTO

O LA CESAREA

\begin{tabular}{|l|r|r|}
\multicolumn{2}{c}{$n$} & \multirow{2}{*}{ Ninguna } \\
\hline Perineal & 3.396 & 42.0 \\
\hline Peridural & 2.411 & 29.7 \\
\hline General & 1.063 & 13.1 \\
\hline Pudenda & 873 & 10.8 \\
\hline Raquided & 314 & 3.9 \\
\hline No INF0 & 31 & 0.4 \\
\hline En & 13 & 0.1 \\
\hline
\end{tabular}

\section{Anestesia para el parto o la cesárea}

El $42 \%$ de los casos no recibió anestesia, una tercera parte $(30 \%)$ recibió anestesia perineal y el resto anestesia general o peridural (en proporciones comparables) para la práctica de operaciones ceśareas.

Cuadro No. 22

FORMA DE TERMINACION

DEL EMBARAZO

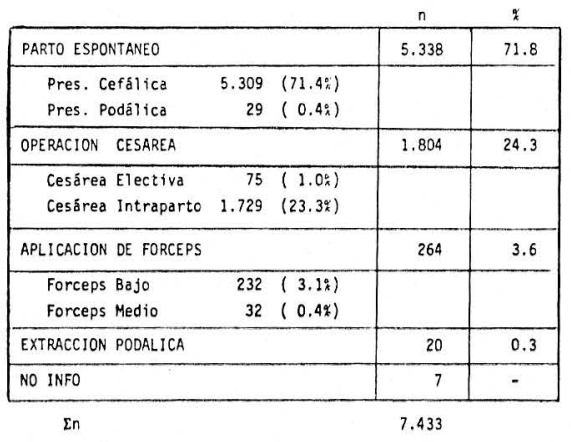

\section{Forma de terminación del embarazo}

El 76\% de los casos tuvo parto vaginal con muy bajas proporciones de aplicación de forceps y de extracción podálica.

El 24\% terminó en operación cesárea lo cual representa un índice muy alto si se le compara con los pocos datos disponibles en algunos años anteriores. En términos muy generales puede decirse que la práctica de la operación cesárea ha ido aumentando desde $(8 \%)$ hace 20 años hasta las cifras actuales. Es innegable que la inserción de algunas tecnologías de monitorización materna y fetal ha inducido un aumento del parto intervenido. En este parámetro es absolutamente indispensable estudiar el impacto del procedimiento sobre la mortalidad perinatal y sobre el estado del recién nacido, cruzando los datos obtenidos en este estudio. Hasta el momento no tenemos ninguna información sobre aspectos tan fundamentales como los enumerados atrás, a pesar de que el incremento mayor de la operación cesárea se dio hace ya 6 años.

\section{Sexo del recién nacido}

Llama la atención una alta proporción de falta de información en un dato tan obvio como éste. Posiblemente se deba a falta de registro en los recién nacidos por operación cesárea.

\section{Tiempo entre la rotura de membranas y el parto}

En menos de la mitad de los casos (44\%) el lapso entre la rotura y la ex-

Cuadro No. 23

SEXO DEL RECIEN NACIDO

\begin{tabular}{|l|c|c|}
\multicolumn{2}{c}{$n$} & $\%$ \\
\hline Femenino & 3.641 & 45.9 \\
\hline Masculino & 3.953 & 49.8 \\
\hline NO INFO & 336 & 4.3 \\
\hline$\Sigma n$ & 7.930 &
\end{tabular}


Cuadro No. 25

DIAS DE HOSPITALIZACION

\begin{tabular}{|l|l|l|l|l|l|}
\hline \multicolumn{2}{|c|}{$0-1$} & \multicolumn{2}{|c|}{$2-3$} & $<4$ & \\
\hline$n$ & $\%$ & $n$ & $\%$ & $n$ & $\%$ \\
\hline
\end{tabular}

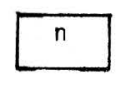

\begin{tabular}{|l|}
\hline Parto \\
\hline Cesárea \\
\hline Aborto \\
\hline E. Ectópico \\
\hline
\end{tabular}

\begin{tabular}{|c|c|c|c|c|c|}
\hline 3.890 & 66.7 & 1.675 & 28.7 & 264 & 4.6 \\
\hline 144 & 8.0 & 1.348 & 74.8 & 311 & 17.2 \\
\hline 193 & 35.4 & 285 & 52.0 & 70 & 12.8 \\
\hline 11 & 11.1 & 74 & 74.7 & 14 & 14.2 \\
\hline
\end{tabular}

\begin{tabular}{|r|}
\hline 5.829 \\
\hline 1.803 \\
\hline 548 \\
\hline 99 \\
\hline
\end{tabular}

Cuadro No. 24

TIEMPO ENTRE LA ROTURA DE LAS MEMBRANAS Y EL PARTO

\begin{tabular}{|c|c|c|}
\hline HORAS & $n$ & $*$ \\
\hline$<1$ & 3.372 & 44.0 \\
\hline $2-5$ & 1.184 & 15.5 \\
\hline $6-23$ & 1.496 & 19.5 \\
\hline 224 & 502 & 6.5 \\
\hline NO INF0 & 1.104 & 14.5 \\
\hline$\sum n$ & 7.658 & \\
\hline
\end{tabular}

pulsión del feto fue igual o menor a una hora, lo cual reduce las posibilidades de complicaciones fetales.

\section{Dias de hospitalización}

En términos generales se observan hospitalizaciones muy cortas para el parto $y$ un poco mayores para los procedimientos quirúrgicos.

\section{Indice de Apgar}

La proporción de recién nacidos deprimidos (Apgar $<6$ al minuto de vida) es muy a'ta $(12 \%)$. En 4 centros asistenciales de Medellín es $7.5 \%$ y en una maternidad de Buenos Aires 8\%, utilizan. do el mismo modelo de historia clínica.
A los 5 minutos de vida la proporción de niños deprimidos sigue siendo alta (3.35\%) si se le compara con los mismos centros $(1.7 \%$ y $2 \%$ respectivamente). $(2,3)$.

\section{Atención inmediata del recién nacido}

El $60 \%$ de los partos fueron atendidos por internos $(43 \%)$, estudiantes $(14 \%) \mathrm{V}$ auxiliares de enfermería (2\%). Los residentes de Obstetricia y Pediatría atendieron el $27 \%$ de los mismos, cifra sospechosamente comparable a la de operaciones cesáreas. Se impone estudiar los resultados perinatales de estos grupos ya que una hipótesis razonable es que nuestras elevadas proporciones de depresión neonatal y de mortalidad perinatal pueden estar relacionadas con este tipo de atención. No parece lógico que una

\section{Cuadro No. 26}

INDICE DE APGAR

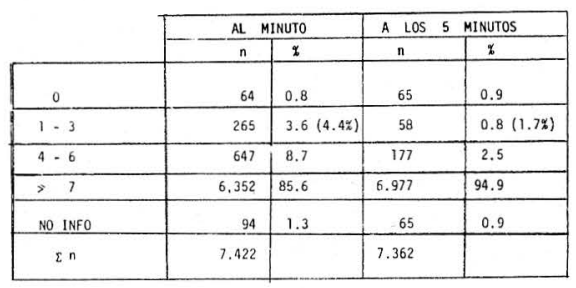


población que muestra tan elevadas tasas de patología obstétrica sea atendida básicamente por personal en formación de pregrado.

Cuadro No. 27

ATENCION INMEDIATA DEL NEONATO

\begin{tabular}{|c|c|c|}
\hline interno & 3.215 & 43.4 \\
\hline Ke'sidente de Obstetricia y Ginecologia & 1.122 & 15.1 \\
\hline Istudiante & 1.050 & 14.2 \\
\hline Residente de Pediatria & 873 & 11.8 \\
\hline Médico de Planta & 652 & 8.8 \\
\hline Lifermera & 202 & 2.7 \\
\hline Auxiliar de Enfermería & 174 & 2.3 \\
\hline Aries tesiólogo & 28 & 0.5 \\
\hline No Info & 92 & 1.2 \\
\hline
\end{tabular}

\section{Peso al nacer}

En la parte inferior del cuadro se ha destacado la tasa de niños de bajo peso al nacer por 100 nacidos vivos. Se observa cómo los niños con peso $<2.500$ gramos constituyen el $14 \%$ de la población atendida. En un estudio realizado con este mismo modelo, en 59 maternidades de 11 países de América Latina, sólo una informa tasas ligeramente superiores a la nuestra (en Brasil, 14.8\% nacidos vivos). Las 58 restantes represen$\tan$ tasas que oscilan entre $13.9 \%$ y $4.6 \%$ con un promedio de 9 recién nacidos de bajo peso por 100 nacidos vivos. (5).

Se sabe que el bajo peso al nacer constituye el 78\% de la mortalidadneonatal precoz en las maternidades de América Latina. (5) Esto obliga a estudiar con precisión las relaciones que existen en nuestro medio, entre este parámetro y la mortalidad perinatal y plantea como indispensable el programa de detención del parto prematuro dentro del Hospital y de rastreo de los factores de riesgo de parto prematuro en los centros periféricos de Cali.
Cuadro No. 28

PESO AL NACER (gr)

PESO AL NACER $(g r)$

\begin{tabular}{|l|c|c|}
\hline \multicolumn{1}{c}{} & $n$ & $\%$ \\
\hline $1.001-1.500$ & 57 & 0.8 \\
\hline $1.501-2.000$ & 131 & 1.8 \\
\hline $2.001-2.500$ & 248 & 3.4 \\
\hline $2.501-3.000$ & 702 & 9.5 \\
\hline $3.001-3.500$ & 1.996 & 27.1 \\
\hline $3.501-4.000$ & 2.628 & 35.7 \\
\hline $4.001-4.500$ & 1.199 & 16.3 \\
\hline $4.501-5.000$ & 284 & 3.8 \\
\hline$>5.000$ & 32 & 0.4 \\
\hline No Info & 6 & 0.1 \\
\hline
\end{tabular}

in $7.361^{\circ}$

\begin{tabular}{|c|c|c|}
\cline { 2 - 3 } \multicolumn{1}{c|}{} & $<2.500$ & $>2.500$ \\
\hline$n$ & 1.138 & 6.145 \\
\hline$\%$ & 14.1 & 85.9 \\
\hline
\end{tabular}

* por cien nacidos vivos

\section{Patología neonatal}

Las madres, que como vimos atrás presentaron un $79 \%$ de patología durante el embarazo, produjeron recién nacidos con sólo un $24 \%$ de patología. La mayōr proporción correspondió al Sindrome de Dificultad Respiratoria que tiene posibilidades de ser disminuida desarrollando acciones tanto durante el embarazo, como el período neonatal inmediato.

El contraste entre la elevada proporción de patología materna $y$ la relativa baja proporción de patología neonatal, 
descrita atrás, podría explicarse por la existencia de patologías maternas que no afectan agudamente al feto, como por ejemplo: la infección urinaria, la mayoría de las roturas prematuras de membranas $y$ otras. Sin embargo, insistimos en la necesidad de realizar cruces entre la patología materna y el resultado neonatal, para establecer relaciones causales.

Cuadro No. 29

PATOLOGIA NEONATAL

\begin{tabular}{|c|c|c|c|}
\hline & \multirow[b]{2}{*}{$n$} & \multirow[b]{2}{*}{ \% } & \\
\hline & & & \% PAT. MATERNA \\
\hline Con Pdologid & 1.805 & 24.0 & 79.0 \\
\hline Sin Patologia & 5.626 & 76.0 & 21.0 \\
\hline in & 7.431 & & \\
\hline $\begin{array}{l}\text { Sindrome de Dificultad } \\
\text { Respiratoria }\end{array}$ & 524 & & \\
\hline Infección No Resp. & 133 & & \\
\hline Anomal 1 fas Concén 1 tas & 105 & & \\
\hline Isoinitunización Rh & 98 & & \\
\hline Hiperbili irrubinemia & 82 & & \\
\hline Pos tnadurez & 17 & & \\
\hline Hemorraq ia Intracraneana & 7 & & \\
\hline Pat. Neurolóqica & 5 & & \\
\hline sn & 1.053 & & \\
\hline OTRAS & 1.431 & & \\
\hline $2 n$ & 2.484 & & \\
\hline
\end{tabular}

\section{Mortalidad}

La mortalidad materna fue de 4.5 por mil nacidos vivos, cifra muy alta, aún tratándose, como se trata, de mortalidad institucional. Para comparación, se dan los datos de la década del 60 , o sea hace 20 años, de la mortalidad materna en el Instituto Materno Infantil de Bogotá. Estos datos fueron calculados por nosotros tomando en cuenta el número de nacidos vivos y las muertes maternas informadas cada año.

$1960=4.5$ por mil nacidos vivos
$1961=2.7$ por mil nacidos vivos
$1962=2.1$ por mil nacidos vivos
$1963=2.3$ por mil nacidos vivos
$1964=2.0$ por mil nacidos vivos

Gráfico No. 3

PESO AL NACER

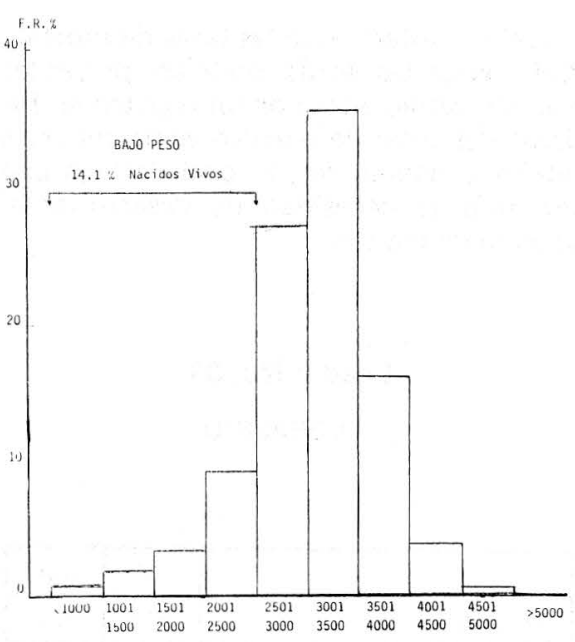

La mortalidad fetal fue de 40.1 por mil nacidos vivos, también muy alta si se la compara con la misma institución, la cual fluctuó en el mismo lapso de 1960 a 1965 entre 31 y 33 por mil nacidos vivos.

La mortalidad neonatal "precoz", definiendo como tal aquella que ocurre durante el período de hospitalización neonatal, fue de 20.4 por mil nacidos vivos. Desafortunadamente, resulta casi imposible tener una cifra de mortalidad

Cuadro No. 30

MORTALIDAD

\begin{tabular}{|l|c|c|}
\hline Materna & 36 & $4.5 \% \%$ \\
\hline Fetal & 322 & $40.1 \% \%$ \\
\hline Neonatal "Precoz" & 164 & $20.4 \%$ \\
\hline
\end{tabular}

ndcidos vivos : 8.027 
neonatal, ya que no disponemos de los datos de sobrevivencia de los recién nacidos durante el primer mes de vida.

Debe anotarse que las tasas de mortalidad expuestas atrás podrían presentar algunas variaciones por subregistro en los datos del total de nacidos vivos, muertes fetales $y$ neonatales, lo cual destaca una vez más la necesidad de desarrollar el sistema de registro.

\section{Cuadro No. 31}

PUERPERIO

\begin{tabular}{|l|r|r|}
\multicolumn{2}{c}{$n$} & \multicolumn{2}{c|}{$n$} \\
\hline Normal & 7.254 & 92.6 \\
\hline Infección & 252 & 3.2 \\
\hline Hemorragia & 170 & 2.2 \\
\hline Otras & 158 & 2.0 \\
\hline No INF0 & 2 & - \\
\hline$x n$ & 7836 & \\
\hline
\end{tabular}

Las cifras descritas en este cuadro corresponden a lo que usualmente se describe en Hospitales Universitarios de países subdesarrollados.

\section{COMENTARIOS}

1. Casi 30 años después de iniciadas las actividades académicas del Departamento de Obstetricia y Ginecología, se establece el uso de una historia clínica perinatal y un programa destinado a sistematizar toda la información médica. Este instrumento parece fundamental para:
a) adecuar la oferta institucional de salud, a la demanda;
b) ejercer auditoría médica y,

c) actualizar permanentemente las conductas vigentes.

2. El bajo nivel socioeconómico de la población atendida, así como su elevada proporción de patología y las altas tasas de mortalidad, operación cesárea y depresión del recién nacido, nos permiten concluir que en las actuales condiciones no es posible atender adecuadamente a las pacientes que nos son referidas.

3. Se hace indispensable mejorar mucho más los sistemas de registro $\mathrm{y}$ de análisis de los datos.

\section{RECOMENDACIONES}

1. Escribir normas de atención médica para todas las entidades encontradas con este programa, priorizando aquellas que presentan la mayor proporción. Deberán distribuirse tanto a los estudiantes de pregrado, como a los de post-grado.

2. Todo el profesorado con asignaciones en las Secciones de Obstetricia y de Cuidado Intensivo Neonatal debe supervisar diariamente y con responsabilidad horaria prefijada, la confècción de la Historia Clínica Perinatal.

3. Es necesaria la contratación de una persona con preparación formal en estadística médica que dirija la labor de las auxiliares de registro médico, para lograr la más alta calidad en la obtención y reducción de la Historia Clínica Perinatal.

4. El análisis de los datos puede hacerse manualmente, como se ha hecho en este trabajo. Sin embargo, no es aconsejable continuar esta práctica, debido al excesivo tiempo que requiere y a las limitaciones de exploración analítica que impone la gran cantidad de datos. 
5. Por esta razón, se hace indispensable la asesoría de personal capacitado en informática tanto para participar en el montaje del programa de computación, como para supervisar los resultados.

6. La totalidad de los datos deberán ser informados con los cruces respectivos, con perioricidad mensual. Cada año se hará una publicación interna comparada, de diagnóstico de situación perinatal.

7. Con periodicidad semanal se discutirán los casos de morbimortalidad evitable con el personal involucrado y se variarán las normas que lo ameriten.

8. Se propone modificar el horario del profesorado, de tal forma que pueda brindar supervisión directa a estudiantes, internos y residentes, durante las 24 horas diarias. Sin una modificación estructural de esta magnitud no podría adquirirse el compromiso de bajar las tasas de morbimortalidad institucional.

9. Se impone realizar el mismo esfuerzo de ordenamiento en las áreas ginecológicas de los Hospitales Universitarios.

10. Todo el proceso unificado de información deberá salir hacia la periferia de los Hospitales Universitarios y abarcar los 3 niveles de atención médica que el país está desarrollando.

\section{REFERENCIAS}

1. BELIZAN, J.M., DIAZ, A.G., GIACOMINI, H., HORCHER, R. MARTELL, M., ONETO, M., QUARANTA, P., Y SCHWARCZ, R.L., Historia Cínica Perinatal. Propuesta de un modelo. Ministerio de Bienestar Social. Rep. Argentina y Organización Panam. de la Salud (OPS). 1976.

\section{Agradecimientos}

Se agradece la colaboración del Dr. Armando González, Director General del Hospital Universitario del Valle en el periodo que cubre este estudio, así como la del personal docente del Departamento de Obstetricia y Ginecología y la de nuestros estudiantes de pre y postgrado, sin cuyo aporte diario, no hubiera sido posible la realización de esta fase del trabajo.

\section{RESUMEN}

Se presentan los datos obtenidos mediante la implantación de un modelo de Historia Clínica Perinatal y la realización manual de su análisis, en 8.181 pacientes hospitalizadas en el Departamento de Obstetricia y Ginecología del Hospital Universitario del Valle. Este artículo es solo descriptivo y por ello solo permite algunas pocas conclusiones y recomendaciones, expuestas al final del mismo.

\section{SUMMARY}

Basic clinical data obtained by using a precodified model for clinical records, are presented. Manual data retrieval for more than three million data corresponding to 8.181 patients admitted to the Obstetrical ward of the University Hospital, in Cali, were performed. This paper deals with a descriptive analysis of the above mentioned data.

2. DIAZ, A.G. FESCINA, R.H., COLLIA., F.C. URMAN, J., SCHWARCZ, R., GARCIA BERTONE, A., GRASSIS, M., LEZAMA, C., RAMIREZ, M., ILLIA, R., PANAL, M., HORCHNER, R. y TENZER, S.M. Diagnóstico de Situación Perinatal. Publicación Cient ífica No. 846. CLAP-OPS/OMS. Montevideo, 1979. 
3. JUBIZ, A., CARDONA, L.E., RESTREPO de RAMIREZ, L., MANOTAS R.J., HALPERT D., MURILLO, O., CALLEJAS, J.M., SA. LAZAR, J.C., SIERRA, A. y CASAS, N. Diagnóstico de Situación Perinatal. XIV Congr. Col. Obstet-Ginecol. Cartagena. 1981.

4. JUBIZ. A. Embarazo Gemelar. Rev. Col. Obstet-Ginecol. 35: 193, 1984.
5. SCHWARCZ, R., DIAZ, A.G., FESCINA, R, DIAZ ROSELLO, J.L., BELITZKY, R. Y MARTELL, M. El bajo peso al nacer $y$ la mortalidad perinatal en maternidades de América Latina. Publicación Científica No. 975. CLAP-OPS/OMS. Montevideo, 1982. 